\title{
Recent Trends in Patient Registries for Health Services Research
}

\author{
Jürgen Stausberg ${ }^{1}$ Sonja Harkener ${ }^{1} \quad$ Sebastian C. Semler ${ }^{2}$ \\ ${ }^{1}$ Institute for Medical Informatics, Biometry and Epidemiology \\ (IMIBE), Faculty of Medicine, University Duisburg-Essen, Essen, \\ Nordrhein-Westfalen, Germany \\ 2 TMF Technology, Methods, and Infrastructure for Networked \\ Address for correspondence Jürgen Stausberg, MD, PhD, Institute for \\ Medical Informatics, Biometry and Epidemiology (IMIBE), University \\ Hospital Essen, University Duisburg-Essen, Hufelandstr. 55, D-45122 \\ Essen, Germany (e-mail: stausberg@ekmed.de).
} Medical Research, Berlin, Germany

Methods Inf Med 2021;60:e1-e8.

\begin{abstract}
Keywords

- health services research

- patient-reported outcome measures

- patients

- registries

- trends
\end{abstract}

Background Patient registries are an established methodology in health services research. Since more than 150 years, registries collect information concerning groups of similar patients to answer research questions. Elaborated recommendations about an appropriate development and an efficient operation of registries are available. However, the scene changes rapidly.

Objectives The aim of the study is to describe current trends in registry research for health services research.

Methods Registries developed within a German funding scheme for model registries in health services research were analyzed. The observations were compared with recent recommendations of the Agency for Healthcare Research and Quality (AHRQ) on registries in the 21 st century.

Results Analyzing six registries from the funding scheme revealed the following trends: recruiting healthy individuals, representing familial or other interpersonal relationships, recording of patient-reported experiences or outcomes, accepting participants as study sites, active informing of participants, integrating the registry with other data collections, and transferring data from the registry to electronic patient records. This list partly complies with the issues discussed by the AHRQ. The AHRQ structured its ideas in five chapters, increasing focus on the patient, engaging patients as partners, digital health and patient registries, direct-to-patient registry, and registry networks.

Conclusion For the near future, it can be said that the concept and the design of a registry should place the patient in the center. Registries will be increasingly linked together and interconnected with other data collections. New challenges arise regarding the management of data quality and the interpretation of results from less controlled settings. Here, further research related to the methodology of registries is needed. received

September 8, 2020 accepted after revision December 19, 2020 published online April 16, 2021
DOI https://doi.org/

$10.1055 / \mathrm{s}-0041-1724104$ ISSN 0026-1270. (c) 2021. The Author(s).

This is an open access article published by Thieme under the terms of the Creative Commons Attribution-NonDerivative-NonCommercial-License, permitting copying and reproduction so long as the original work is given appropriate credit. Contents may not be used for commercial purposes, or adapted, remixed, transformed or built upon. (https://creativecommons.org/ licenses/by-nc-nd/4.0/)

Georg Thieme Verlag KG, Rüdigerstraße 14, 70469 Stuttgart, Germany 


\section{Introduction}

Patient registries are an established methodology to answer research questions related to a group of similar individuals. ${ }^{1}$ One might argue that the operation of a registry is no longer a scientific challenge today. Surprisingly, this is not true. Application areas and settings of patient registries change rapidly now. ${ }^{2,3}$ For example, registries are increasingly established to support the benefit assessment and the post-marketing surveillance of drugs and medical devices. Objective of this work is to support further development of patient registries for health services research by describing recent trends from the perspective of a German funding scheme.

\section{History and Current State}

The methodology of patient registries evolved over more than 150 years ago, beginning with the National Leprosy Registry of Norway in $1856 .{ }^{4}$ Eleven years later, Alexander von Winiwarter started with his collection of cancer cases in Germany. ${ }^{5}$ The first epidemiologic cancer registry in Germany was established in Hamburg 1926 (cf. https://www.hamburg.de/krebsregister/4330184/krebsregistrierung/). In the year 1974, the World Health Organization summed up the use of registries in epidemiological and clinical research. ${ }^{6}$ Since that time, registries found their way into the broad area of health services research aiming at a description, understanding, and evaluation of daily health care (cf. - Fig. 1). For example, countless registries analyzing the quality of care in relation to types or clusters of health care providers, regions, and even individuals were established. Especially the Nordic countries demonstrate a nearly complete coverage of the population by registries. $^{7}$

Consequently, the state of the art of patient registries was described several times. Newton and Garner reviewed information of 117 out of 234 registries for their report on disease registries in England in 2002. ${ }^{8}$ For Australia, 28 clinical registries concerned with the quality of care were analyzed in 2009. ${ }^{9}$ Beyond a national level, Niederländer et al reported on registries of implantable medical devices in $2013 .{ }^{10}$ However, information about registries is still highly fragmented. As far as the authors know, registries of registries do not exist, neither on a national nor on an international level. The US-American approach suffers from the financial shortages of the Agency for Healthcare Research and Quality (AHRQ https://www.ahrq. gov/ropr/). The European Cross-Border Patient Registries Initiative PARENT left a website with dummy text (http://parentror.eu/). ${ }^{11}$

Nevertheless, high-quality recommendations for the development and the operation of patient registries are available. The AHRQ publishes the most elaborated one with the user's guide about registries for evaluating patient outcomes. Starting with the first version in 2007, the current third edition dates from 2014. ${ }^{12}$ The German Network Health Services Research recently published an update of its Memorandum Registry for Health Services Research originating from $2010 .^{13}$ The recommendations agree that patient registries are complex systems serving predetermined purposes. To address these purposes, registries collect uniform data using a digital infrastructure. The development of patient registries should follow a systematic approach with a planning and design stage. ${ }^{14}$ It is documented in a registry protocol. The operation of a registry includes the employment of staff, the maintenance of rooms and equipment, the communication with study sites and other stakeholders, to name only a few relevant tasks. The individuals within a registry share the same disease (e.g., cancer), take the same drug (e.g., a biosimilar), needed the same procedure (e.g., a liver transplantation), or got the same device (e.g., a pacemaker).

\section{Future Development}

To keep pace with the upcoming requirements, the AHRQ published an addendum for 21 st century patient registries in $2018 .^{15}$ The German Federal Ministry of Education and Research opened a funding scheme for model registries in health services research in 2016. As part of this funding scheme, an accompanying project was launched to support registries within the funding scheme, to organize counseling by external experts for the registries, and to foster the dissemination, transfer, and implementation of the results in registry-based research. The accompanying project is performed in cooperation between the TMF Technology, Methods, and Infrastructure for Networked Medical Research and the Institute for Medical Informatics, Biometry and Epidemiology (IMIBE) at the University of Duisburg-Essen. The TMF is the umbrella organization for networked medical research in Germany (cf. http://www.tmfev.de/). It is the platform for interdisciplinary exchange as well as cross-project and cross-location cooperation to identify and solve organizational, legal, and ethical as well as technological issues.

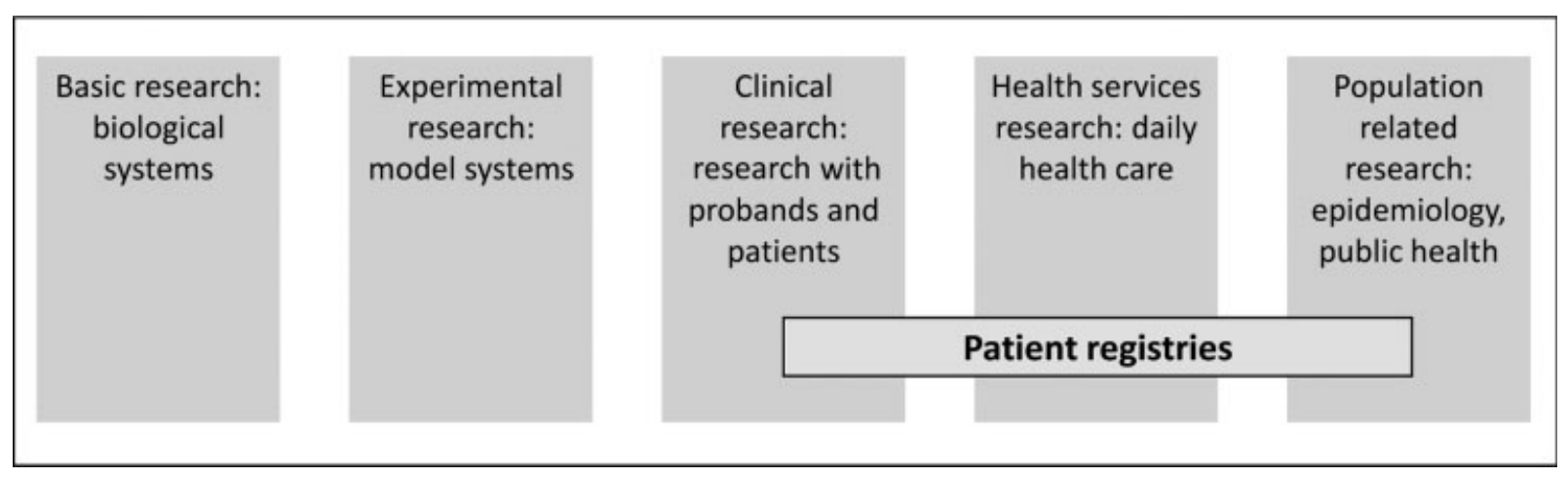

Fig. 1 Use of patient registries in the five areas of medical research. 


\section{Objectives}

The aim of the study is to describe current trends in registry research from the perspective of the accompanying project of the German funding scheme for model registries in health services research and to compare the observations with the view of the AHRQ publishing the most elaborated recommendations concerning registry research.

\section{Materials and Methods}

The German funding scheme for model registries in health services research was split up into two phases. In phase I, 16 projects developed a concept for a registry defined in a registry protocol. ${ }^{16}$ Fourteen out of the 16 projects submitted a proposal for phase II, the realization of the registries. Finally, six projects were selected for this phase (cf. https://www.tmfev.de/EnglishSite/News/articleType/ArticleView/articleId/ 4433.aspx). All projects started in spring 2019, five of them will be funded for 5 years, one for only 3 years (cf. https://www. gesundheitsforschung-bmbf.de/de/modellhafte-register-realisierungsphase-9011.php in German). - Table 1 shows the medical fields of the projects from both phases of the funding scheme. The total funding sum is approximately 13.5 million EURO, excluding the funding of the accompanying project. The accompanying project was established for 2 years and extended for three additional years until autumn 2022.

The six registries of phase II cover a wide range of health conditions on a national level. The legislative basis for all registries is a voluntary participation based on an informed

Table 1 Medical fields in the German funding scheme

\begin{tabular}{|l|l|l|}
\hline Domain & Registry & Phase II \\
\hline Acute conditions & $\begin{array}{l}\text { Acute respiratory } \\
\text { distress syndrome }\end{array}$ & \\
\hline & Fever & $\mathrm{x}$ \\
\hline & Heart attack & \\
\hline & Pulmonary embolism & \\
\hline Chronic diseases & $\begin{array}{l}\text { Recurrent calculus of the } \\
\text { upper urinary tract }\end{array}$ & $\mathrm{x}$ \\
\hline & Noninfectious uveitis & $\mathrm{x}$ \\
\hline & Paraplegia & \\
\hline Rare diseases & Prader-Willi syndrome & $\mathrm{x}$ \\
\hline & $\begin{array}{l}\text { Systemic lupus } \\
\text { erythematosus }\end{array}$ & \\
\hline Oncology & $\begin{array}{l}\text { Hereditary breast and } \\
\text { ovarian cancer }\end{array}$ & $\mathrm{x}$ \\
\hline & Metastasizing breast cancer & \\
\hline Interventions & Knee joint endoprosthesis & \\
\hline & Vaccination & $\mathrm{x}$ \\
\hline $\begin{array}{l}\text { Other } \\
\text { conditions }\end{array}$ & Death & \\
\hline & Living donors & \\
\hline
\end{tabular}

consent. All registries record data electronically using different kinds of electronic data capture systems.

- The registry on Treatment Exit Options for Non-Infectious Uveitis (TOFU) recruits patients suffering from a noninfectious uveitis, a relatively rare chronic disease. A noninfectious uveitis can lead to blindness. A long-term therapy is needed to prevent the progression of the disease. The registry is especially interested in exit options from the immunotherapy (https://www.tofu-uveitis-register.de/). The registry's aim is to describe routine health care.

- RECUR establishes a registry for recurrent calculi of the upper urinary system (https://www.recur-register.de/). About $5 \%$ of the population suffer from those calculi, 2.5\% from recurrent calculi. An acute episode is very painful and accompanied with an admission to a hospital. This registry implements one of the use cases of the German Medical Informatics Initiative, ${ }^{17}$ in particular the MIRACUM Consortium. The registry's aims are to describe routine health care and to gain epidemiologic knowledge.

- HerediCaRe is a cancer registry concerned with hereditary breast and ovarian tumors. Special interest is on genetic subgroups that represent special risks for the course of the disease and for the response of available therapies. Aim of the registry is the evaluation and optimization of a riskadapted prevention strategy.

- ParaReg analyzes alternative pathways in the rehabilitation of paraplegic patients. Alternative pathways occur due to different sectors in Germany offering rehabilitation support, e.g., the sickness funds or the statutory accident insurance (https://www.klinikum.uni-heidelberg.de/zentrum-fuer-orthopaedie-unfallchirurgie-und-paraplegiologie/klinik-fuer-paraplegiologie-querschnittzentrum/forsc hung/sektion-neurorehabilitation/parareg-ein-web-basier tes-register-zum-lebenslangen-monitoring-von-querschnittgelaehmten). The registry's aim is to describe routine health care.

- The FeverApp registry offers parents support in case of feverish children. Additionally, the parents can document the domestic processes and procedures (https://www. feverapp.de/). The data of all participating families are anonymously stored in a central database; a subgroup is controlled by pediatrician practices. The behavior of the families in case of feverish children is compared with evidence-based recommendations from available guidelines. The registry's aims are quality assurance and effectiveness research.

- SOLKID-GNR implements a national registry for the safety of living kidney donors (https://www.lebendspenderegister. $\mathrm{de} /$ ). The results should help to identify organ donors with higher risks of lower quality of life and long-term complications. The registry's aim is to describe routine health care.

ParaReg and SOLKID-GNR strive for case completeness whereas the other registries defined numeric aims for the recruitment rate, partially based on a sample size estimation. With the exception of HerediCaRe (10 years), the registries plan for an unlimited period. Mainly, hospitals are responsible for the recording of data; some registries recruit via 
centers specialized in the respective disease in Germany. Management of data protection and data privacy is documented in corresponding concepts and ensured by data protection and data privacy policies. Responsible ethic commissions had formally acknowledged all projects.

To identify trends, freely available information about the funded projects was used on one hand. On the other hand, the accompanying project met with representatives of the registries between December 2019 and July 2020 to discuss specific and exemplary characteristics of the registries' concepts.

\section{Results}

\section{Recruiting Healthy Individuals}

The term "patient registry" indicates sick individuals as an observable unit suffering from a clearly defined disease or health-related status. This holds true for patients with paraplegia, with recurrent calculus of the upper urinary track, and with noninfectious uveitis. This is not applicable for the remaining three registries. HerediCaRe registers not only cancer patients but also women under risk of cancer or relatives of cancer patients independent of their risk status. The FeverApp registry recruits parents to observe familial behaviors, parents that will never suffer from the symptom "fever in children" the registry is interested in. Living donors underwent a medical procedure that is not intended to diagnose, heal, mitigate, or prevent a status of the same individual. Living donors are "healthy" in respect of the procedure. Of course, the procedure as well as the reduced organ capacity carries a relevant risk that makes living donors "patient like" from the perspective of health care. Contrary to a patient-an individual in contact with the health care system having a medical label like a diseasehealth care providers do not take care of healthy individuals. Therefore, recruitment of observational units via health care providers will not work for healthy individuals in the same way as for patients. Registries have to find new ways to approach those groups of individuals, e.g., by offering selfrecruitment over the web or by contacting them from third parties.

\section{Representing Familial or Other Interpersonal Relationships}

Typically, registries regard patients as independent from each other. There is not any need to represent relationships between observational units. Device-, drug-, or procedureoriented registries are slightly different, because the interesting event (i.e., the observational unit in those registries) might occur multiple times, e.g., a replacement of the hip at the right and left extremity. Those registries have to assign more than one event to an individual patient. Nevertheless, also individuals have interesting relationships with each other. HerediCaRe strives to represent the pedigree of families with members suspect of suffering from hereditary cancer. The FeverApp registry bounds together parents, grandparents, or other caretakers with children using a family identifier. Moreover, the role of the family members is recorded and maintained. Different from the representation of a family tree, SOLKID-GNR is interested in donors and recipients of transplants. Here, the procedure or the organ establishes the interpersonal relationship. As a result, the complexity of a registry increases enormously. Answers towards research questions can depend on the particular role in a relationship or family. It will be impossible to interpret the registries' results without understanding this complexity. Furthermore, not all members of the relationship will be contacted directly. For example, an index woman suffering from breast cancer will be asked for her family anamnesis. If the information of the family anamnesis is not necessary for the individual care of the woman, the family members have to be informed about the inquiry according to the General Data Protection Regulation of the European Union (EU). On one hand, those members might refuse the storage of the information in the registry, on the other, women suffering from breast cancer might disagree with the disclosure of cancer to all family members. Twin research could be a model for the representation of familial or other interpersonal relationships in registries. ${ }^{18,19}$

\section{Recording of Patient-Reported Experiences or Outcomes}

Patient-reported experience measures (PREMs) and patientreported outcome measures (PROMs) are hot topics in medical research ${ }^{20}$. For some time now, they have even been required by National Health Systems (NHS) for quality assessment. ${ }^{21}$ By design, the patients themselves need the right to record data, differently to outcomes and experiences reported by health professionals. Although recording of PREMs and PROMs on paper is possible, the projects of the funding scheme use digital devices. TOFU sends weblinks to the patients for the acquisition of those data. SOLKID-GNR provides tablet computers within the study facilities. RECUR and the FeverApp registry use smartphone apps. In case of the FeverApp registry, the app is already available in the most common app stores. Applying mobile devices and smartphone apps in registry research still suffers from practical problems such as maintenance, security, and safety issues. ${ }^{22}$ Comparability in the use of different technologies (paperbased, smartphone, tablet, desktop) must also be considered. However, the uncontrolled and less structured recording process is the most challenging issue that could lead to low quality and therefore useless data. Source data are missing, thus an onsite monitoring is not possible. A central monitoring process that queries implausible or missing data in the registry's database lacks a feedback channel to the patients. Once recorded, the data are fix. Plausibility checks are the sole option if they are integrated in the software frontend. In summary, the validity of PREMs and PROMs is a critical data quality leak. ${ }^{23}$

\section{Accepting Participants As Study Sites}

The caretakers in the FeverApp registry recruit their families themselves by downloading the app and registering the app in the registry office. The responsibility for the informed consent of the children-depending on age and stage of 
development-is up to the caretakers. Consequently, not only health care professionals can be seen as study sites, having responsibilities for observational units they add to the registry on the one hand and getting feedback concerning data quality and medical issues the registry is concerned with on the other hand. Then, registry owners have to set up a customer relationship management concerning design and operational issues not only to health care professionals that are partially trained in scientific reasoning but also to laypersons that miss any scientific background.

\section{Active Informing of Participants}

The scientific community is the main target group of registries. The science communication further includes politicians, sickness funds, pharmaceutical companies, manufacturers of medical devices, and the interested public. Study sites receive feedback on data quality and clinical performance. The FeverApp registry goes one-step further and offers generic medical information to the caretakers of feverish children. This information addresses diagnostic, therapeutic, and monitoring issues supporting the caretakers in tackling with the current problem. Advice is given about warning symptoms that would require contact to health professionals. However, individual advice would require a certification of the app according to the EU Regulation on Medical Devices. This would raise costs and responsibilities. The active informing of the participants is therefore an unusual service of registries today.

\section{Integrating the Registry with Other Data Collections} RECUR uses the data integration centers established at university clinics within the German Medical Informatics Initiative. ${ }^{17}$ Even in the registry, the data remains locally at the study sites. Just in time, the data are analyzed locally, and the results combined centrally according to the research question. Additional data defined by the registry, i.e., PREMs and PROMs, are stored locally at the study sites as well. HerediCaRe is part of the manifold cancer registry area including the tumor documentation from health care providers, regional clinical cancer registries, federal epidemiologic registries, and the statewide German Centre for Cancer Registry Data at the Robert Koch Institute. The cancer domain is an example of a vertical integration of registries that implements data transfer bottom-up and top-down. Bottom-up, data are reduced from the most possible detail for individual care in the tumor documentation to public needs in the surveillance of cancer epidemiology. Top-down, specific information is provided that may not be available at the individual health care provider, such as vital status or the causes of death (cf. - Fig. 2). SOLKID-GNR strives for a horizontal integration with the upcoming national registry of transplantations. The exchange of data concerning the characteristics of donors-provided by SOLKID-GNR-with the outcome of recipients-available in the national transplantation registry-will be of value for both registries. TOFU intends another variant of a horizontal integration. Whereas TOFU focuses on the noninfectious uveitis, the German Ophthalmologic Society implements a registry covering a broader range of diseases of the eye. Independently of the chosen approach, the integration of registries with other data collection is still a challenge. ${ }^{24}$ Individuals, events, topographic sites (e.g., left and right eye), and all relevant objects have to be unambiguously and consistently identified in all collections. Standard terminologies, common value sets, and metadata conventions have to be available to assure semantic and syntactic interoperability. Participants' and stakeholders' rights have to be controlled according to the General Data Protection Regulation independent of the single registry. Unfortunately, none of the mentioned requirements is in place in Germany. Therefore, registries have to implement individual solutions. For example, all projects in this funding scheme use their own informed consent as legislative basis for the recording of data from individuals. Identifiable data are separated from the medical data and supervised for each registry by a different organizational unit. Each project uses different algorithms and

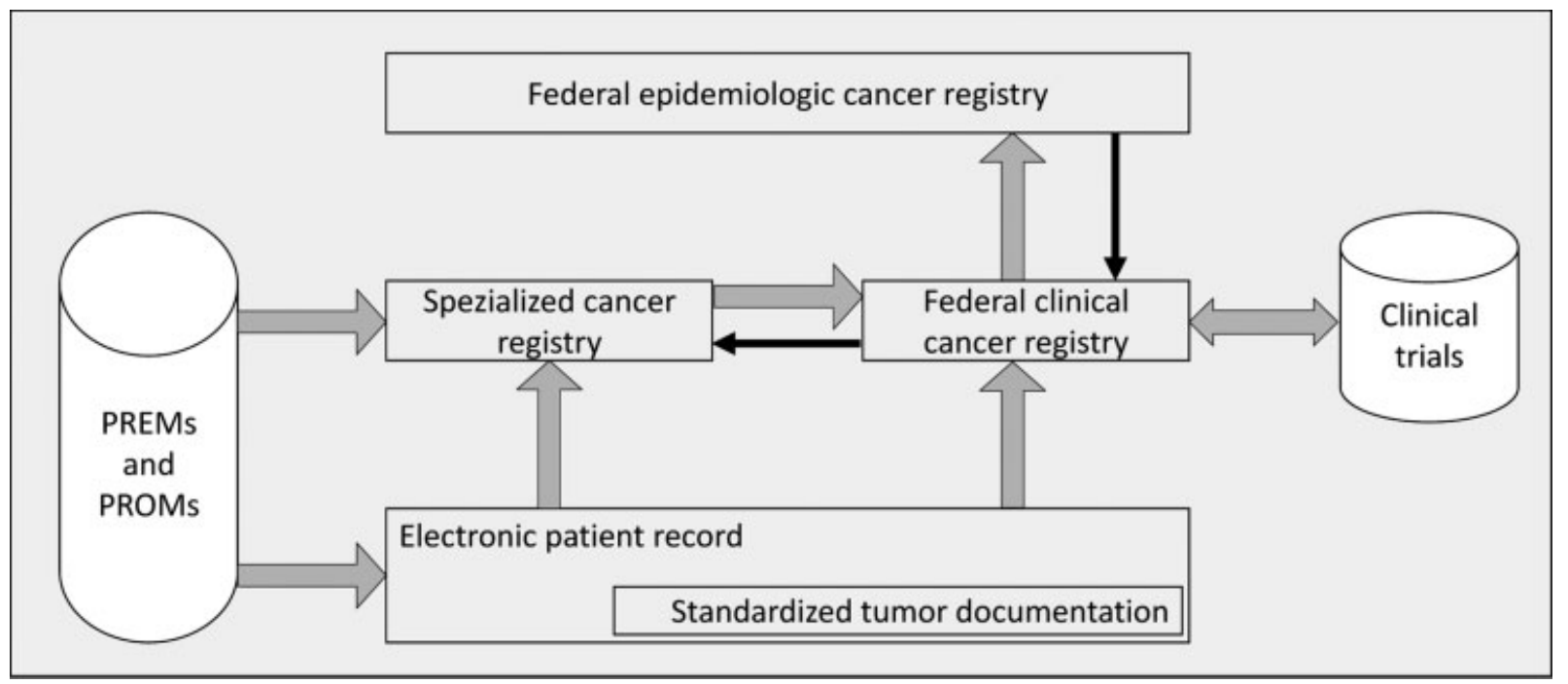

Fig. 2 Vertical integration of registries, the example of cancer. Thick arrows denote a possible transfer of cancer-related data, thin arrows denote information such as the life status of a patient. 
procedures to replace personally identifiable information fields by an artificial identifier, leading to different pseudonyms in each registry for the same individual.

\section{Transferring Data from the Registry to Electronic Patient Records}

Registry data are usually copied from primary data available in paper-based or electronic patient records. With PROMs and PREMs, data not available in the files of health care providers are solely and primarily recorded for the registry. Using its app, RECUR imports data from the patients into the hospital information systems of the participating centers. Similarly, the data recorded by the families in the FeverApp could be transferred to the pediatricians in charge of the children and stored in the local IT systems. Then, the pediatricians might use the data to improve the quality of care they offer to the sick children.

\section{Discussion}

Four of the seven trends identified from the German funding scheme address a closer relationship between the registry owners and the observational units that are recruited for registries, usually patients but in case of the FeverApp registry, for example, families. Looking at the IT infrastructure, none of the registries implement an uncommon solution. Putting patients and citizens in the center seems to be the major driver for new and challenging concepts for registries.

This observation is in accordance with the AHRQ's view on 21st Century Patient Registries. ${ }^{15}$ The view is split-up in five chapters:

1. Increasing Focus on the Patient

2. Engaging Patients as Partners

3. Digital Health and Patient Registries

4. Direct-to-Patient Registry

5. Registry Networks

Here, three of the five chapters focus on the patient. However, some differences exist between the presented trends and the AHRQ's view. Patient and public involvement had been already pointed out as important success factors. ${ }^{7}$ Claiming for a patient-centered research design in point 1 , the AHRQ goes far beyond the current state. For example, a patient-centered research design would incorporate the patient perspective from the early beginning, e.g., the determination of the research questions. This task still seems to be in professional hands in Germany. The presented observations support the notion of patients as partners. Especially the recording of PREMs and PROMs requires an active participation of patients in registries, changing their role from an observational unit to a customer or even to a stakeholder. Furthermore, on a technological level, apps and other interfaces become more important means to acquire patient-reported data. Patient registries are becoming part of digital health, additionally exemplified by RECUR that makes use of universities' data integration centers. One step further, a registry might be established virtually based on electronic health records. ${ }^{25}$ Engaging patients as members of a registry's executive committee is still in its infancy. A prominent example for a registry operated by a self-help group is the German Cystic Fibrosis Registry. ${ }^{26}$ The necessity for vertical or horizontal integration supports the demand for registry networks, either on a technical as well as on a strategical level. However, the infrastructure is not in place to support this necessity. In Germany, a national service for the identification of individuals in research projects is missing. Therefore, research projects have to define and implement proprietary solutions if they intend to share registry data about individuals recorded on the basis of an informed consent. An integration of data that had not been considered from the beginning is nearly impossible yet. Even the identification of running registries is difficult due to the lack of a registry of registries in United States as well as in Germany. Therefore, it is hard to find out which other registries could be approached to build up a network. Self-recruitment is an important function of direct-to-patient registries. The FeverApp registry implements self-recruitment as well as active informing of the families about the interesting condition. Eventually, patients will operate registries without any support by health care professionals. PatientsLikeMe could be regarded as a template for such a registry. ${ }^{27}$ However, equating a commercial platform as PatientsLikeMe with an engagement of patients might be questionable. - Table 2 opposes the identified trends with the headings from the AHRQ.

Two trends remain that were not mentioned in the AHRQ's publication: recruiting healthy individuals and representing familial or other interpersonal relationships. Both aspects are interrelated. For example, familial relationships exist between sick and healthy individuals. From this perspective, the concept of a "patient registry" might not be sufficient in the near future. Consequently, recommendations focusing only on patient registries or disease registries will miss those trends. Registries

Table 2 Presented trends and headings from the AHRQ

\begin{tabular}{|c|c|}
\hline Trends & Headings from the AHRQ \\
\hline \multicolumn{2}{|l|}{ Recruiting healthy individuals. } \\
\hline \multicolumn{2}{|l|}{$\begin{array}{l}\text { Representing familial or other } \\
\text { interpersonal relationships. }\end{array}$} \\
\hline $\begin{array}{l}\text { Recording of patient-reported } \\
\text { experiences or outcomes. }\end{array}$ & $\begin{array}{l}\text { Increasing focus on } \\
\text { the patient } \\
\text { Digital health and } \\
\text { patient registries }\end{array}$ \\
\hline $\begin{array}{l}\text { Accepting participants } \\
\text { as study sites. }\end{array}$ & $\begin{array}{l}\text { Engaging patients } \\
\text { as partners } \\
\text { Direct-to-patient registry }\end{array}$ \\
\hline $\begin{array}{l}\text { Active informing } \\
\text { of participants. }\end{array}$ & Direct-to-patient registry \\
\hline $\begin{array}{l}\text { Integrating the registry with } \\
\text { other data collections. }\end{array}$ & $\begin{array}{l}\text { Digital health and } \\
\text { patient registries } \\
\text { Registry networks }\end{array}$ \\
\hline $\begin{array}{l}\text { Transferring data from the } \\
\text { registry to electronic } \\
\text { patient records. }\end{array}$ & $\begin{array}{l}\text { Digital health and } \\
\text { patient registries }\end{array}$ \\
\hline
\end{tabular}

Abbreviation: AHRQ, Agency for Healthcare Research and Quality. 
in the future will be concerned with health-related issues, including healthy individuals, individuals at specific risks, individuals having signs, symptoms, genetic or phenotypic characteristics, or suffering from a disease. Therefore, we suggest replacing the term "patient registry" with the term "health-related registry" to cover the upcoming needs.

\section{Conclusion}

Registries in health care are a dynamic and innovative methodology. The current evidence concerning the optimal field of application, their systematic development, and an efficient operation is summarized in several recommendations from a traditional point of view. However, the methodology does not remain stable. Registry owners are faced with challenges that are not addressed in the recommendations yet. Setting-up specific funding schemes that accompany those innovative projects could be an appropriate reaction. Until updated recommendations are available, the new registries have to be utilized with caution. Established indicators of data quality like case completeness, data completeness, and validity have to be analyzed differently. ${ }^{28}$ Especially patient-reported data and self-recruitment are out of control of registries' offices. It is nearly impossible to check whether recorded outcomes occured in reality, if the patients themselves recorded the outcomes using a smartphone app. Probably, registries in the 21 st century will suffer from a lower data quality because of their innovative design. Therefore, a standardized reporting scheme describing data quality would be essential to assess whether research questions could be appropriately answered with a data collection at hand. ${ }^{29}$ The interpretation of the new world registries would be challenging as well. Observational units could include patients suffering from a disease or having a special condition who are in contact with the health care system just as healthy people not being in contact with the health care system at all. The same events are reported several times from different perspectives, e.g., by health care providers, affected individuals, and relatives or other third parties. ${ }^{30}$ Data are merged within a multifold scene of data collection, each following its own rules and regulations. The concept of patient registries, as defined by the AHRQ, will merge with data repositories as loose collection of data from arbitrary sources.

Based on the findings of the study, we believe that it would be worthwhile to develop a new taxonomy of observational study types to avoid a chaotic collection of data samples. Then the term "registry" could be preserved for clearly defined projects that follow a registry protocol with predefined research questions and a strict data management policy. Concepts have to be worked out that capture extensions of registries that follow the presented trends but loose strength of the fundamental methodology. Only then, recipients would be able to assess opportunities and risks of scientific results based on knowledge about strength and weaknesses of study types.
Funding

The project was funded by the German Federal Ministry of Education and Research under contracts 01GY1720A/ 01GY1720B and 01GY1917A/01GY1917B. The authors gratefully acknowledge the cooperation with the six registries.

Conflict of Interest

None declared.

\section{References}

1 Dreyer NA, Garner S. Registries for robust evidence. JAMA 2009; 302(07):790-791

2 Fleurence RL, Blake K, Shuren J. The future of registries in the era of real-world evidence for medical devices. JAMA Cardiol 2019;4 (03):197-198

3 Lauer MS, D'Agostino RB Sr. The randomized registry trial-the next disruptive technology in clinical research? N Engl J Med 2013;369(17):1579-1581

4 Irgens LM. The origin of registry-based medical research and care. Acta Neurol Scand Suppl 2012;126(Suppl 195):4-6

5 Winiwarter AV. Cancer (1848-1916). 1948:356. Available at: https:// acsjournals.onlinelibrary.wiley.com/toc/10970142/1948/1/3

6 Brooke EM. The Current and Future Use of Registers in Health Information Systems. Geneva: World Health Organization; 1974

7 Jervelund SS, de Montgomery CJ. Nordic registry data (part III): use of healthcare services, complications hereof, and inequalities in mortality. Scand J Public Health 2020;48(03):241-242

8 Newton J, Garner S. Disease Registers in England. Oxford: Institute of Health Sciences, University of Oxford; 2002

9 Evans SM, Bohensky M, Cameron PA, McNeil J. A survey of Australian clinical registries: can quality of care be measured? Intern Med J 2011;41(1a):42-48

10 Niederländer C, Wahlster P, Kriza C, Kolominsky-Rabas P. Registries of implantable medical devices in Europe. Health Policy 2013;113(1-2):20-37

11 Zaletel M, Kralj M, eds. Methodological Guidelines and Recommendations for Efficient and Rational Governance of Patient Registries. Ljubljana: National Institute of Public Health, Slovenia; 2015

12 Gliklich R, Dreyer N, Leavy Me. Registries for Evaluating Patient Outcomes: A User's Guide. 3rd ed. Two Volumes. Rockville, MD: Agency for Healthcare Research and Quality; 2014

13 Stausberg J, Maier B, Bestehorn K, et al. Memorandum registry for health services research: update 2019. Gesundheitswesen 2020; 82(03):e39-e66

14 Leiner F, Haux R. Systematic planning of clinical documentation. Methods Inf Med 1996;35(01):25-34

15 Gliklich RE, Dreyer NA, Leavy MB, Christian JB, eds. 21st Century Patient Registries. Ebook Addendum to Registries for Evaluating Patient Outcomes: A User's Guide 3rd ed. Rockville, MD: Agency for Healthcare Research and Quality; 2018

16 Stausberg J, Harkener S, Siddiqui R, Semler SC. IT infrastructure for registries in health services research: a market study in Germany. Stud Health Technol Inform 2018;251:183-186

17 Semler SC, Wissing F, Heyder R. German medical informatics initiative. Methods Inf Med 2018;57(S 01):e50-e56

18 Moayyeri A, Hammond CJ, Hart DJ, Spector TD. The UK adult twin registry (TwinsUK Resource). Twin Res Hum Genet 2013;16(01): 144-149

19 Skytthe A, Kyvik KO, Holm NV, Christensen K. The Danish Twin Registry. Scand J Public Health 2011;39(Suppl 7):75-78

20 Nilsson E, Orwelius L, Kristenson M. Patient-reported outcomes in the Swedish national quality registers. J Intern Med 2016;279 (02):141-153 


\section{e8 Recent Trends in Patient Registries Stausberg et al.}

21 Timmins N. NHS goes to the PROMS. BMJ 2008;336 (7659):1464-1465

22 Coons SJ, Eremenco S, Lundy JJ, O'Donohoe P, O'Gorman H, Malizia $\mathrm{W}$. Capturing patient-reported outcome (PRO) data electronically: the past, present, and promise of ePRO measurement in clinical trials. Patient 2015;8(04):301-309

23 Chang EM, Gillespie EF, Shaverdian N. Truthfulness in patientreported outcomes: factors affecting patients' responses and impact on data quality. Patient Relat Outcome Meas 2019;10:171-186

24 Mears GD, Rosamond WD, Lohmeier C, et al. A link to improve stroke patient care: a successful linkage between a statewide emergency medical services data system and a stroke registry. Acad Emerg Med 2010;17(12):1398-1404

25 Findlay I, Morris T, Zhang R, et al. Linking hospital patient records for suspected or established acute coronary syndrome in a complex secondary care system: a proof-of-concept e-registry in National Health Service Scotland. Eur Heart J Qual Care Clin Outcomes 2018;4(03):155-167
26 Prinz N, Zolin A, Konrad K, et al; ECFS patient registry and the DPV registry. Characteristics of cystic fibrosis-related diabetes: data from two different sources the European cystic fibrosis society patient registry and German/Austrian diabetes prospective follow-up registry. Pediatr Diabetes 2019;20(03): 255-262

27 Wicks P, Massagli M, Frost J, et al. Sharing health data for better outcomes on PatientsLikeMe. J Med Internet Res 2010;12(02):e19

28 Harkener S, Stausberg J, Hagel C, Siddiqui R. Towards a core set of indicators for data quality of registries. Stud Health Technol Inform 2019;267:39-45

29 Rajan NS, Gouripeddi R, Mo P, Madsen RK, Facelli JC. Towards a content agnostic computable knowledge repository for data quality assessment. Comput Methods Programs Biomed 2019; 177:193-201

30 Eichler GS, Cochin E, Han J, et al. Exploring concordance of patientreported information on PatientsLikeMe and medical claims data at the patient level. J Med Internet Res 2016;18(05):e110 